\title{
Preparation of Linear Low-density Polyethylene/Treated Date Palm Leaflet Green Composites and Investigation of their Thermal and Mechanical Properties
}

\author{
MASHAEL ALSHABANAT* \\ Chemistry Department, Science College, Princess Nourah bint Abdulrahman University, Riyadh, \\ Kingdom Saudi Arabia. \\ ${ }^{*}$ Corresponding author E-mail: ma.naif@ hotmail.com \\ http://dx.doi.org/10.13005/ojc/350123
}

(Received: March 24, 2018; Accepted: December 06, 2018)

\begin{abstract}
This work aims to develop green linear low-density polyethylene (LLDPE) composites that are commercially viable due to the low cost of the date palm leaflet filler, which is a local renewable resource. The filler was naturally treated with gum arabic solution. FT-IR, XRD, and SEM techniques were used to characterize the samples. The thermal and mechanical properties were measured by TGA, DSC, and tensile testing. The results showed noticeable changes in the properties of the composites compared to those of the original LLDPE sample. TGA revealed TGA revealed that the composite started thermally. The composites started thermally degrading before the original polymer, owing to the degradation of the natural components in the filler. The findings from DSC suggested that the crystallinity was affected. The tensile testing results indicated that the composites were appropriate for applications requiring low tensile strength at break and high Young's modulus. A comparison of these results with earlier ones exhibited that the basic additives in the polymer may have an effect on the filler performance.
\end{abstract}

Keywords: Green composites, Date palm Leaflet, Linear low-density polyethylene, Mechanical properties, Thermal properties.

\section{INTRODUCTION}

Polymeric materials find widespread application due to their useful properties such as light weight. In the past few decades, the use of fillers to enhance the polymer properties and/or reduce cost has become increasingly popular. From an environmental and economical perspective, agricultural waste has attracted the attention of researchers as a natural and renewable resource. Therefore, several studies on polymer bio-composites have been conducted ${ }^{1-5}$. However, because of the diversity of the natural materials available by geographic area, it is still important to research polymer bio-composites based on locally sourced materials. In this context, and for application purpose, the investigation of their properties and characterize them is an important work in the research field.

This is an Open Access article licensed under a Creative Commons license: Attribution 4.0 International (CC- BY). Published by Oriental Scientific Publishing Company @ 2018 
In Saudi Arabia, different types of polymers are produced, one of which is linear low-density polyethylene (LLDPE). Which is manufactured locally in various grades for different applications, including furniture pads, packaging, and garden products. The date palm tree is also important in Saudi Arabia, both economically and environmentally. Optimal utilization of the waste of date palm tree is a good strategy for realizing a sustainable environment.

Although date palm waste is often used as a polymer filler ${ }^{6-10}$, the novel part of this work is the development of a new design to prepare/treat this waste for use as a filler. The filler treatment process is essential to ensure adhesion between a hydrophobic non-polar polymer and a hydrophilic polar filler. Chemical, physical, and mechanical treatment processes have been applied and published in the literature ${ }^{11-14}$. However, it should be noted that chemical treatments focus on using chemicals, which may be environmentally harmful.

In our earlier work ${ }^{15}$, we developed a green treatment process to naturally treat date palm waste with a gum arabic (GA) solution, which, to the best of our knowledge, was not reported before, and investigated the viscosity, and thermal and mechanical properties of the LLDPE/treated leaflet composites. In this work, we aimed to apply the same preparation conditions, recycle the naturally treated leaflet as a filler for preparing inexpensive and ecofriendly composites of LLDPE, and analyze the thermal and mechanical properties, taking into consideration that the target grade of the LLDPE in this study differs from that of the previous study on basic additives and applications, to investigate the effect of the filler with different types of basic additives on the final properties of the LLDPE composites.

\section{MATERIALS AND METHODS}

The polymeric matrix used in the experiments was LLDPE, grade R40039, supplied by SABIC, Saudi Arabia. As this material is UV-stabilized, it is suitable for outdoor applications.

For the filler, date palm tree leaflets were collected from a private local farm, cut, ground, and sieved to obtain fine particles with sizes $\leq 600 \mu \mathrm{m}$. A natural treatment was applied by washing the fine powder with distilled water and then with GA solution using an electric mixer then sonication, filtration, after which the powder was dried in sunlight for a few days.

As described in our previous report ${ }^{15}$, the preparation was performed using a two-roll mill at $190^{\circ} \mathrm{C}$ to prepare $3 \mathrm{~mm}$ thick sheets of the polymer composites and unfilled polymer samples, F-LLDPE and LLDPE. Scanning electron microscopy (SEM, Quanta 250), infrared spectroscopy (FT-IR Perkin-Elmer spectrophotometer), and $X$ ray diffraction (Bruker AXS Gmbh, D8 Advance) were performed to characterize the samples.

The thermal and mechanical properties of the composites were evaluated by thermogravimetric analysis (TGA), differential scanning calorimetry (DSC), and tensile testing using a Perkin-Elmer thermal analysis system, Universal V4.5A TA instrument, and Zwick/Roell tester, respectively. TGA was performed under a nitrogen atmosphere at a heating rate of $10^{\circ} \mathrm{C} / \mathrm{min}$, from 30 to $800^{\circ} \mathrm{C}$. The DSC heating scans ranged from 30 to $200^{\circ} \mathrm{C}$ with sample weights of $\sim 2 \mathrm{mg}$. The tensile testing was performed according to ASTM D 638 standards.

\section{RESULTS AND DISCUSSION}

The FT-IR spectra of LLDPE and F-LLDPE are illustrated in Fig. 1. Table1 shows the spectral data of the absorption bands for the LLDPE and composite spectra. Some shifts, especially a considerable one in the absorption band of LLDPE at $2836 \mathrm{~cm}^{-1}$, which shifted to $2825 \mathrm{~cm}^{-1}$, indicated interactions between the polymer chain and filler particles.

Table 1: FT-IR and F-LLDPE

\begin{tabular}{|c|c|c|}
\hline LLDPE & F-LLDPE & $\begin{array}{l}\text { Functional group and } \\
\text { type of vibration }\end{array}$ \\
\hline Frequency $\left(\mathrm{cm}^{-1}\right)$ & Frequency $\left(\mathrm{cm}^{-1}\right)$ & \\
\hline 2961.11 & $\begin{array}{l}2963.24 \\
\text { (broad band) }\end{array}$ & $\mathrm{CH}$ stretched vibration ${ }^{16}$ \\
\hline 2836.86 & 2825.58 & \\
\hline 1464.55 & 1463.12 & $\begin{array}{l}\mathrm{CH}_{2} \text { and } \mathrm{CH}_{3} \text { bending } \\
\text { vibration }{ }^{16}\end{array}$ \\
\hline 1366.90 & 1364.39 & $\mathrm{CH}_{3}$ Symmetric \\
\hline 1303.81 & 1303.96 & $\mathrm{CH}$ skeletal vibration ${ }^{16}$ \\
\hline
\end{tabular}




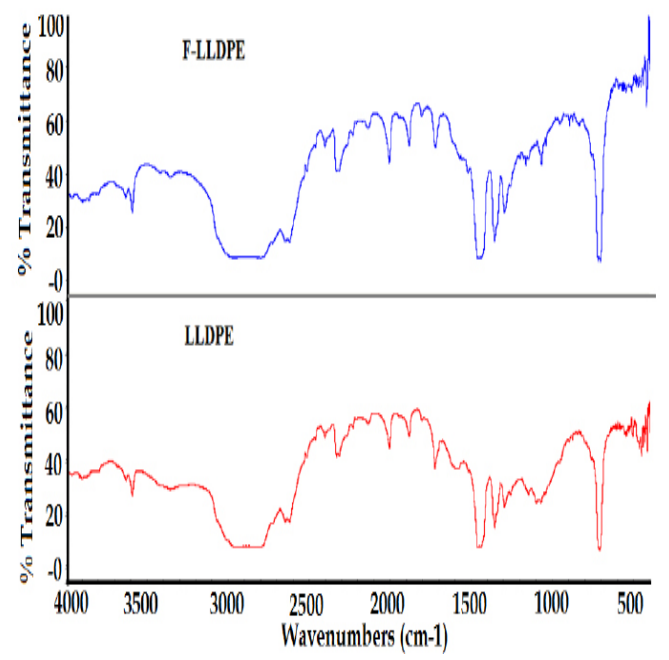

Fig. 1. FT-IR spectra of LLDPE and F-LLDPE

The XRD patterns of LLDPE and F-LLDPE and the relevant data are shown in Table 2 and Fig. 2, respectively. From the data, the unit cell of the LLDPE crystal was determined to be orthorhombic ${ }^{14}$ cited in ${ }^{1}$. The XRD spectrum of F-LLDPE was similar to that of LLDPE, with similar angles and d-spacing, except for changes in the intensity of the peaks. The variation in the intensity indicates that the change in the crystal habit supports this interpretation, as do some previously published results ${ }^{17}$. According to Inoue and Hirasawa ${ }^{17}$, the intensity of each diffraction peak is related to the crystal habit and not to the crystal shape, where the crystal habit results from "the difference in the growth rate of each crystal face."

Table 2:XRD data of LLDPE and F-LLDPE

\begin{tabular}{|c|c|c|c|c|c|}
\hline $2 \theta(\stackrel{\circ}{)})$ & $\begin{array}{c}\text { LLDPE } \\
\text { d-spacing }(\AA)\end{array}$ & Intensity & $2 \theta(\stackrel{\circ}{)})$ & $\begin{array}{c}\text { F-LLDPE } \\
\text { d-spacing }(\AA)\end{array}$ & Intensity \\
\hline 21.286 & 4.17072 & 39898 & 21.303 & 4.16756 & 30198 \\
\hline 23.642 & 3.76020 & 8675 & 23.660 & 3.75736 & 7226 \\
\hline 36.014 & 2.49179 & 1043 & 36.031 & 2.49064 & 662 \\
\hline
\end{tabular}

Figure 3 presents the SEM images of untreated (a) and treated leaflets (b and c), while Fig. 4 presents the SEM images of LLDPE and F-LLDPE. It was clear from Fig. 3 that the treated filler (b) showed distortions in the leaflet surface as compared to the surface of the untreated sample (a). These distortions could act as active bonding sites between the filler particles and the polymer chains. There were obvious variations in the particle size and irregular shapes were seen in the treated filler, as shown in Fig. 3 (c). This variation affected the distribution of the filler particles within the matrix, and consequently, the final properties of the composites. Furthermore, from Fig. 4, the LLDPE sample appeared smooth, whereas the F-LLDPE sample appeared rough due to the filler particle distribution within the matrix.
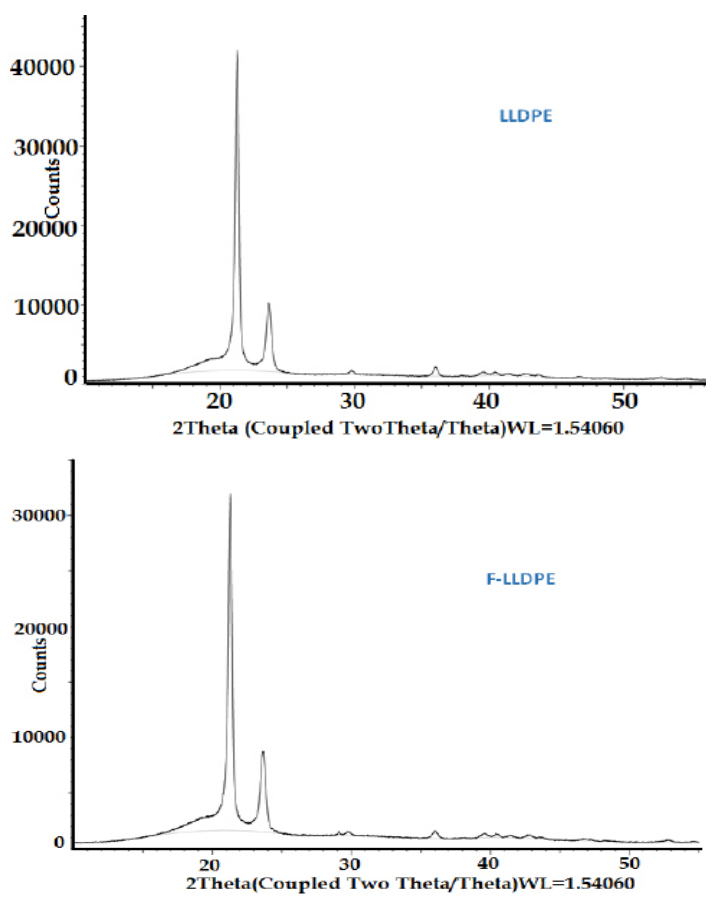

Fig. 2. XRD patterns of LLDPE and F-LLDPE
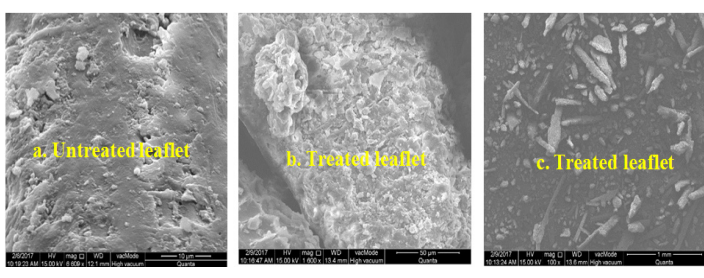

Fig. 3. SEM images of untreated (a) and treated leaflet (b and c)

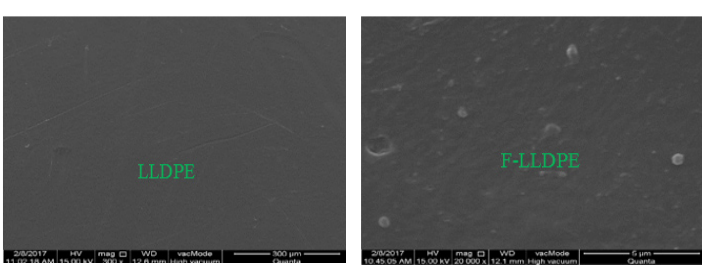

Fig. 4. SEM images of LLDPE and F-LLDPE

To investigate the thermal stability, TGA was performed on the LLDPE and F-LLDPE samples (Fig. 5). The thermal decomposition of both samples occurred in one stage, from $\left(T_{1}\right) 333^{\circ} \mathrm{C}$ to $\left(T_{2}\right) 468^{\circ} \mathrm{C}$ for the LLDPE samples and from $\left(T_{1}\right)$ $300^{\circ} \mathrm{C}$ to $\left(T_{2}\right) \sim 467^{\circ} \mathrm{C}$ for the F-LLDPE composite. 
This decomposition could be attributed to the disintegration of the $\mathrm{C}-\mathrm{C}$ bonds in the main chain'. However, the $T_{1}$ of the F-LLDPE sample decreased as compared to that of the LLDPE sample, which could be due to the loss of the natural component in the filler. Yang et al., ${ }^{18}$ reported that hemicellulose, cellulose, and lignin decomposed in the temperature ranges $220-315^{\circ} \mathrm{C}, 315-400^{\circ} \mathrm{C}$, and $160-900^{\circ} \mathrm{C}^{18}$, respectively, (see Figure 6).

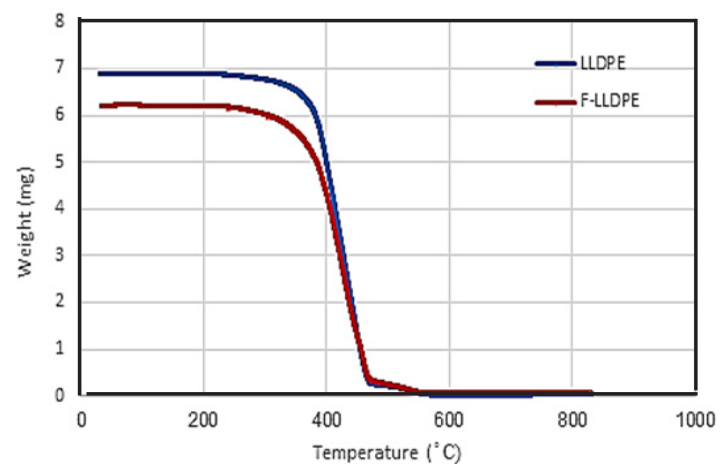

Fig. 5. TGA curves of LLDPE and F-LLDPE samples

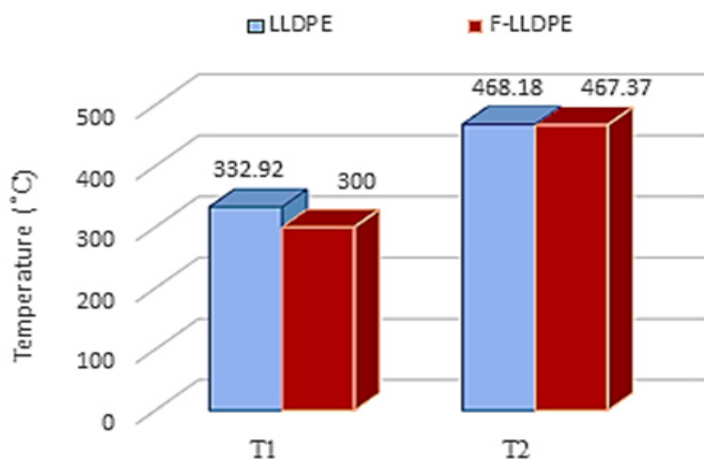

Fig. 6. Comparison the onset $T\left(T_{1}\right)$ and endset $T\left(T_{2}\right)$ of LLDPE and F-LLDPE

The DSC curves of LLDPE and F-LLDPE are shown in Fig. 7. A single peak appeared at $104.82^{\circ} \mathrm{C}$ in the LLDPE curve, which could be attributed to an exothermal transition transition $\left(T_{c}\right)$ with an enthalpy $\left(\Delta H_{c}\right)$ of $110.6 \mathrm{~J} / \mathrm{g}$. The peak was shifted to a higher temperature by $\sim 8^{\circ} \mathrm{C}$ in the F-LLDPE curve to $112.05^{\circ} \mathrm{C}$, with a sharp increase in $\Delta H_{m}(181.2 \mathrm{~J} / \mathrm{g})$. Another single peak appeared at $120.80^{\circ} \mathrm{C}$ in the curve of the LLDPE sample, with an enthalpy $\left(\Delta H_{m}\right)$ of $112.6 \mathrm{~J} / \mathrm{g}$, and it was attributed to an endothermic melting transition $\left(T_{m}\right)$. However, this peak slightly shifted to a higher temperature by $\sim 5^{\circ} \mathrm{C}$ in the curve of the F-LLDPE sample and appeared at $125.23^{\circ} \mathrm{C}$ with a significant increase in $\Delta H_{m}(175.2 \mathrm{~J} / \mathrm{g})$. These observations suggest that the addition of the filler affects the crystallinity of LLDPE by affecting the crystal habit during the growth of the polymer crystal, as indicated by the XRD results. Dehghani et al., reported that the crystallinity behavior of date palm leaf fiber/recycled poly(ethylene terephthalate) composite samples was affected, and the DSC analysis indicated an increase in the onset Tc and a higher degree of crystallinity of the composites, demonstrating that date palm leaf fibers could act as nucleating agents, facilitating the crystalline entity formation and increasing the overall crystallinity ${ }^{19}$.
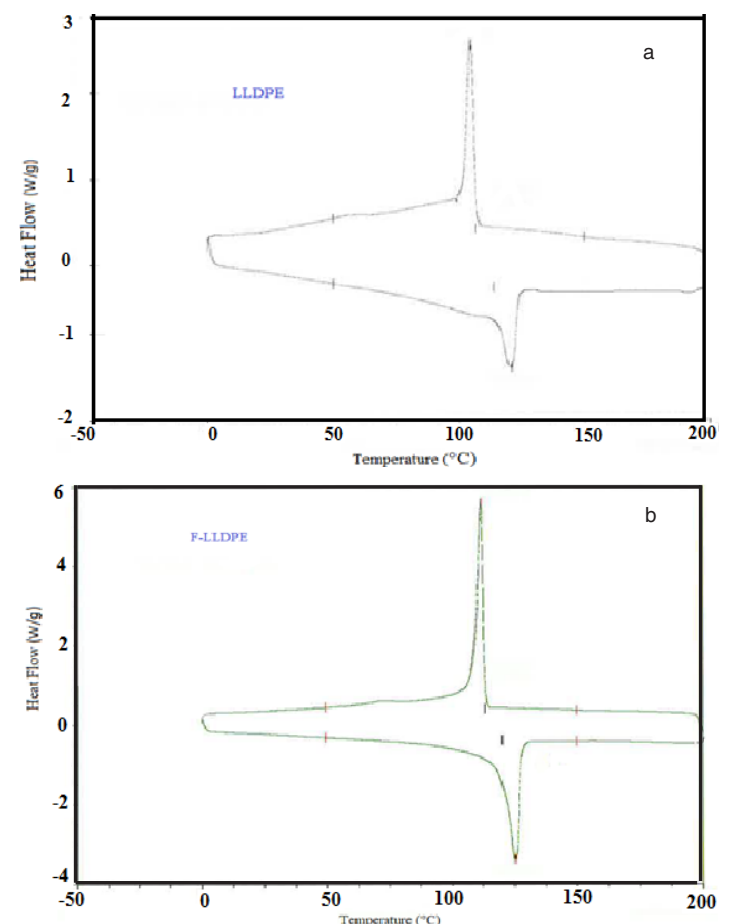

Fig. 7. DSC curves of (a) LLDPE and (b) F-LLDPE

Comparison these results with that of our previous study ${ }^{15}$ is shown in Fig. 8 . Firstly, it is important to clarify again that the grade of LLDPE in this study differs from that in the previous, owing to the different type of basic additive: the former was produced with a UV-stabilizer for outdoor applications while the latter was produced with lubricating additives (anti-block and slip) for packaging applications. The samples of both grades of unfilled LLDPE and their composites were prepared under the same conditions, with the same filler.

It is clear that the $T_{m}, T_{c}, \Delta H_{m}$, and $\Delta H_{c}$ of both grades of unfilled LLDPE were almost the same. However, after adding the treated date 
palm leaflet filler, it was shown that the values of $\mathrm{Tm}, \mathrm{Tc}, \Delta \mathrm{Hm}$, and $\Delta \mathrm{H}_{\mathrm{c}}$ for the composites in the previous study were unaffected, compared to those for the original LLDPE, whereas in this study, their recorded values increased, especially the $\Delta \mathrm{Hm}$ and $\Delta \mathrm{H}_{\mathrm{C}}$, which significantly increased, indicating that the crystallinity was enhanced; this may have been because the date palm leaflet could act as a nucleating agent, as discussed above.

This observation suggested that the type of polymer additive may impact the performance of the treated leaflet as a filler for LLDPE. In this study, the UV-stabilizer additive positively affected the influence of the filler on the crystallinity of the composites, whereas the anti-block and slip additives did not have the same effect; this may have been because of the combined presence of the anti-block and slip additives, or one of them inhibiting the action of the filler as a nucleation agent.

A possible interpretation for these results is that the UV-stabilizer may have contributed to the improvement in the compatibility of the LLDPE/ treated date palm leaflet filler surfaces, leading to an improvement in the dispersibility of the filler into the polymeric matrix, which in turn may have provided more sites for nucleation.

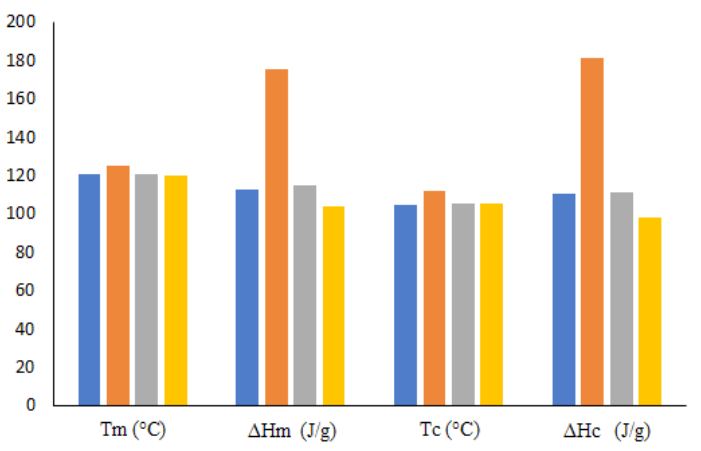

$\backsim$ Present LLDPE $\approx$ Present F-LLDPE $₫$ Previous LLDPE $₫$ Previous F-LLDPE

Fig. 8. A comparison of the DSC data of this study with those of the previous

The tensile break strength, tensile yield, elongation break strength, elongation yield, and Young's modulus were measured to investigate the mechanical properties of the LLDPE and F-LLDPE samples. The results are shown in Fig. 9. There was a significant decrease in the tensile yield, tensile break strength, elongation break strength, and elongation yield, as well as a sharp increase in Young's modulus, upon the addition of the date palm leaflet filler. The reduction in tensile break strength could be attributed to the adhesion between the polymer and the filler ${ }^{10,} 20-22$ cited in10.

Although the purpose of treating the filler is to improve the interfacial adhesion, other factors such as orientation and distribution of the filler particles also affect the interfacial adhesion. The shape and size of particles impact their distribution and orientation throughout the polymeric matrix, which reflects on the interfacial adhesion. Generally, the more uniform the distribution, the better is the adhesion. The SEM images in Fig. 3 reveal irregular shapes and sizes of the particles, which negatively impacted their distribution and orientation in the matrix, and consequently, had a negative effect on the interfacial adhesion. Additionally, the parameters of the tensile testing may be influential, as indicated in some published studies ${ }^{23}$. Furthermore, the type of filler can affect the mechanical properties, and the increase in Young's modulus could be attributed to this factor as the increase in the cellulose content in the natural filler has a positive impact on Young's modulus ${ }^{13}$.

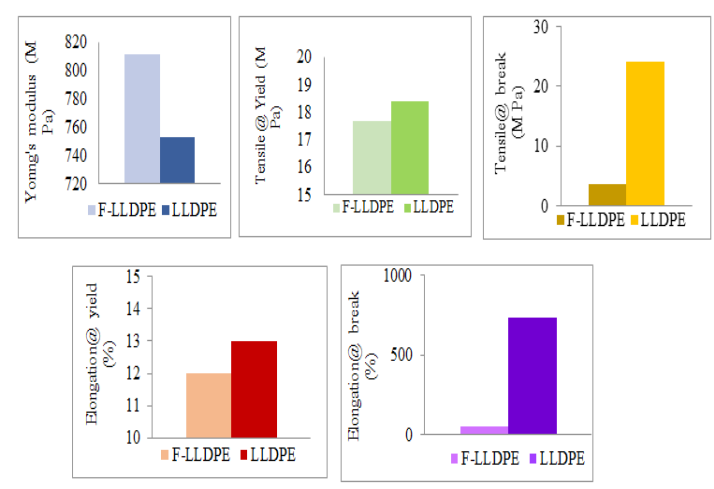

Fig. 9. Mechanical properties of LLDPE and F-LLDPE

Finally, a comparison of the mechanical properties of the current composite samples with those of the previous one ${ }^{15}$ is shown in Fig. 10. The values of the tensile strength at break, elongation at break, tensile strength at yield, elongation at yield, and Young's modulus of both the grades of unfilled LLDPE were different, and this was because they were designed for different applications. Accordingly, the type of basic additive was selected. However, the effect of addition of filler on both the grades seemed to be similar. A decrease in the tensile strength at break and elongation at break, and an increase in the Young's modulus, were notable. There was a slight decrease in the tensile at yield and elongation 
at yield for the current composites, whereas the samples in the previous study did not even record any values of them (both the composite and unfilled polymer). However, the percentage of decrease or increase was different for both the composites. The percentage of the increase in the Young's modulus for the current composites was about $8 \%$, and that for the previous one was $14 \%$, the percentage of decrease in the tensile strength at break and elongation at break for the current composite

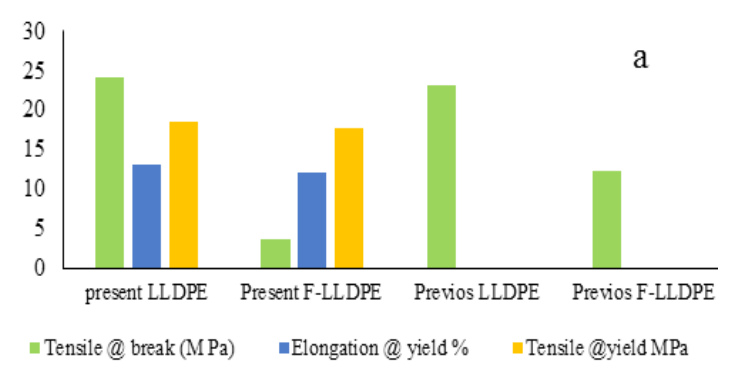

( $80 \%$ and $90 \%$, respectively), and those for the previous one $(50 \%$ and $40 \%$, respectively, and the percentages of the decrease in the tensile at yield and elongation at yield were about $3.5 \%$ and $7.5 \%$, respectively, for the current composites. Although this observation may support our suggestion for effect of the basic additives in the polymer on the filler performance, further investigation is needed to provide more information and gain clarity.

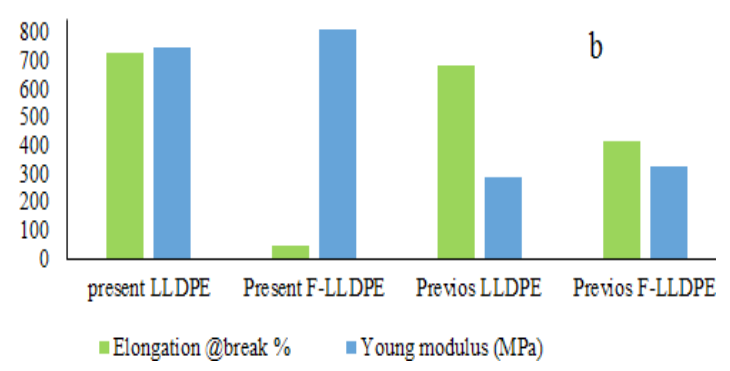

Fig. 10. A comparison of the mechanical data of this study with those of the previous, (a) tensile at break, elongation at yield and tensile at yield, (b) elongation at break and Young's modulus

\section{CONCLUSION}

Polymer bio-composites were prepared using a linear low-density polyethylene (LLDPE) and $5 \mathrm{wt} \%$ of a locally sourced filler made of date tree leaflets. A natural treatment of the filler particles was applied using GA solution. The prepared composites were characterized using different techniques, including FT-IR, XRD, and SEM. The thermal and mechanical properties were measured by TGA, DSC, and tensile testing. The results of these analyses showed that the thermal and mechanical properties were influenced by the addition of the filler. The values of $\mathrm{Tm}, \mathrm{Tc}, \Delta \mathrm{Hm}$, and $\Delta \mathrm{HC}$ notably increased, and the thermal stability decreased.The composites were found to be suitable for applications requiring low tensile strength at break and high Young's modulus. The findings of this study were comparable with those for the LLDPE/naturally treated date palm leaflet composites in our previous work, and they suggested that the type of polymer additive may affect the performance of the filler; however, additional studies to provide more information about the effect of the type of polymer basic additive on the performance of polymer fillers is also required.

\section{ACKNOWLEDGEMENT}

This research did not receive any specific grant from funding agencies in the public, commercial, or not-for-profit sectors

\section{REFERENCES}

1. Shebani, A.N.; Vanreenen, A. J., J Compos Mater., 2009, 43,1305-1318.

2. Sain, S.; Sengupta, S.; Kar, A.; Mukhopadhyay, A.; Sengupta, S.; Kar, T.; Ray, D., Polym Degrad Stab., 2014, 99, 156-165.

3. Kumar, R.; Yakubu, M. K.; Anandjiwala, R. D., Express Polym Lett., 2010, 4, 423-430.

4. Hussein A. Shnawa, Nadum A. Abdulah and Faise J. Mohamad, World Appl Sci J., 2011, 14, 1730-1733.
5. G. U. Raju, S. Kumarappa, V. N., J. Mater. Environ. Sci., 2012, 3(5) 907-916.

6. Alajmi; M., Shalwan, A., Materials., 2015, 8, 4163-4173.

7. Atuanya, C. U.; Government M. R.; NwobiOkoye, C. C.; Onukwuli, O. D., Int J Mech Mater Eng., 2014, 1:7.

8. Ameeh, O.A.; Tijani ISA, M., Leonardo El J Pract Technol., 2015, 26, 65-78.

9. AlMaadeed, M.A.; Nógellová, Z.; Micušík, M.; Novák, I., Krupa, I., Mater. Des., 2014, 53, 29-37. 
10. Mirmehdi, S.M.; Zeinaly, F.; Dabbagha, F., Composites: Part B., 2014, 56, 137-141.

11. Tlijania, M.; Gouadria, A.; Benyounes, R.; Durastanti, JF.; Mazioud, A., Inter. J. Sci.: Basic and Appl. Res., 2013, 11, 30-38.

12. Alawar, A.; Hamed, A.M.; Al-Kaabi, Kh., Composites: Part B., 2009, 40, 601-606.

13. Al-Oqla, F.M.; Alothman, O.Y.; Jawaid, M.; Sapuan S. M.; Es-Saheb M. H., "Biomass and bioenergy processing and properties" Springer International publishing Switzerland, 2014, Chap. 1.

14. Du, W.; Zhong, W.; Lin, Y.; Shen, L.; Du, Q., Europ. Polym. J., 2004, 40, 1987-1995.

15. Alshabanat, M; Inter. J. Chem Tech Res., 2018, 11, 115-124.

16. Mulla M., Ahmed J., Al-Attar H., CastroAguirre Edgar, Arfat Y.A., Auras R., Food
Cont., 2017, 73, 663-671.

17. Inoue, M.; Hirasawa, I., J. Crys. Growth., 2013, 380, 169-175.

18. Yang, H.; Yan, R.; Chen, H..; Lee D.H., Zheng, C., Fuel., 2007, 86, 1781-1788.

19. Alireza Dehghani, Sara Madadi Ardekani, Mariam A. Al-Maadeed, Azman Hassan, Mat Uzir Wahit, Mater. Des., 2013, 52, 841-848.

20. Bledzki, A. K.; Reihmane, S.; Gassan, J., PolymPlastic Technol Eng., 1998, 37, 451-68.

21. Bledzki, A. K., Gassan, J., Prog Polym Sci., 1999, 24, 221-74.

22. Felix, J. M., Gatenholm, P., J Appl Polym Sci, 1991, 42(3), 609-20.

23. Kutty, S. K. N.; Nando, G. B., Plas. Rubber Compos. Process. Appl., 1993, 19, 105-110. 